\title{
Thermal Energy Supply of Residential Districts with Thermal Pumps and Solar Power Stations
}

\author{
Petrosyan A. L. \\ Chair of Ventilation, Gas and Heat Supply \\ National University of Architecture and Construction of Armenia \\ Republic of Armenia
}

\begin{abstract}
Thermal energy supply of residential buildings with the help of thermocompressor heat pumps using solar energy as a low temperature heat source have a great disadvantage of requiring considerable surfaces of solar collectors and electric capacities thus resulting that high-power energy consumption. Within daytime and favorable weather conditions solar power plantserves as a source of electricity generation as it is shown in the suggested technological scheme. In order to increase the energy index of the plant it is recommended to use low evaporating temperature refrigeration agents as operating body which will first be evaporated in solar collectors then will be expanded in turboexpanders. As a result the condensing heat will be used in heat pump evaporator wich will make it possible to combine solar power plant condenser with heat pump evaporator. The process of modification of solar power plant thermal energy conversion efficiency in accordance with the increase of evaporation and overheating temperature in operating body and vacuum reflector solar collector surface size modification for the purpose of supplyingspecific electric powercorresponding 1 kilowatt total heat heat demandin solar power plant turboexpander was considered for climatic conditions in Yerevan. Different types of frons have been considered as operating body. $R 114$ and $R 22$ have better characteristics of which practical application may have $R 22$, since it is actually possible to organize production of low-power and quantity turboexpanders. Since this factor is one of the main and currently the production of turboexpanders with methane and benzobutane is organized in Russia consequently technical capability of application of $R 600 a$ has also been considered. In the proposed scheme during the remaining hours and days of the year when the application of solar power plant is inexpedient it is supposed to use gas cogeneration station which simultaneously provides part of the heat energy requirement of the region.
\end{abstract}

Keywords: residential neighborhoods, heatcool supply, heat pump, solar power station, low - temperature working bodies, optimum temperature conditions

\section{Introduction.}

In order to supply residential buildings with thermal power with the help of heat pumps and solar power plants a low and medium temperature heat source with a certain thermal potential is required. This can be concentrated solar energy of various types of solar collectors. As a consequence, low temperature heat source energy transforms into medium temperature thermal potential heat source in heat pumps which is used for heating separate buildings. In summer heat insulation and heat transfer from buildings serve a low temperature heat source for heat pumps, cold supplying is carried out, they are cooled down, and the condensate heat sink in heat pumps is used to supply hot water. Since heat pump electric motor consumes $1 / 3 \ldots 1 / 4$ of the generated electricity, then solar power plant is included in suggested technological schem, in which low evaporation temperature refrigerants are intended to be used as operating body. It will cause the increase of energy efficiency of solar power plant and heat pump and solar collector surface decrease. The need to apply this version stems from the fact that about $40 \%$ of currently extracted fuel is spent to create artificial microclimate in residential buildings. As a source of heat, cold and electricity supply various types of gas heat exchanger gas boilers with organic fuels usage, electricity and thermal energy consumption steam compressor and absorption bromine-lithium refrigerating machines, heat pumps, as well as mainly electricity and in part thermal energy generating thermal power stations, gas cogeneration stations are used. 
The use of non-traditional technology schemes, such as solar power, is associated with major technical difficulties and financial costs, especially in case of large capacity development. Some part of the generated electricity is spent on electric motors of local and central air conditioning compressors of various heat and cold generation system equipped with steam compressor refrigerating machine and heat pump. In heat pump equipped local and central air conditionings as a low temperature heat source are used subsoil waters, solar power with low temperature solar collectors and solar pools. In parallel with the heat pump studies, solar energy using solar power plants have been monitored aimed at providing heat pump electricity demand by means of non - traditional methods, namely freons, isobutane, butane and etc.

\section{EASE OF USE}

Study results made in accordance with the technological scheme shown in pic. 1 are performed in this article. The joint use of solar power for generating electricity with the use of solar power plant and heat pump is implemented in scheme in order to supply the district with heat and warm water. Besides, heat pump has the ability to realize cold supply in summer. Solar power plants and gas cogeneration stations cohesion gives an apportunity to meet the demand of heat, cold and electricity supply of the district within the year thereby ensuring independence from the overall energy system, but not from fuel in particular from the gas supply system. As it is performed in scheme the heating of solar power plant operating body is carried out gradually in flat solar collectors (19), the evaporation in vacuum solar collector, the overheating in vacuum reflector solar collector (20).

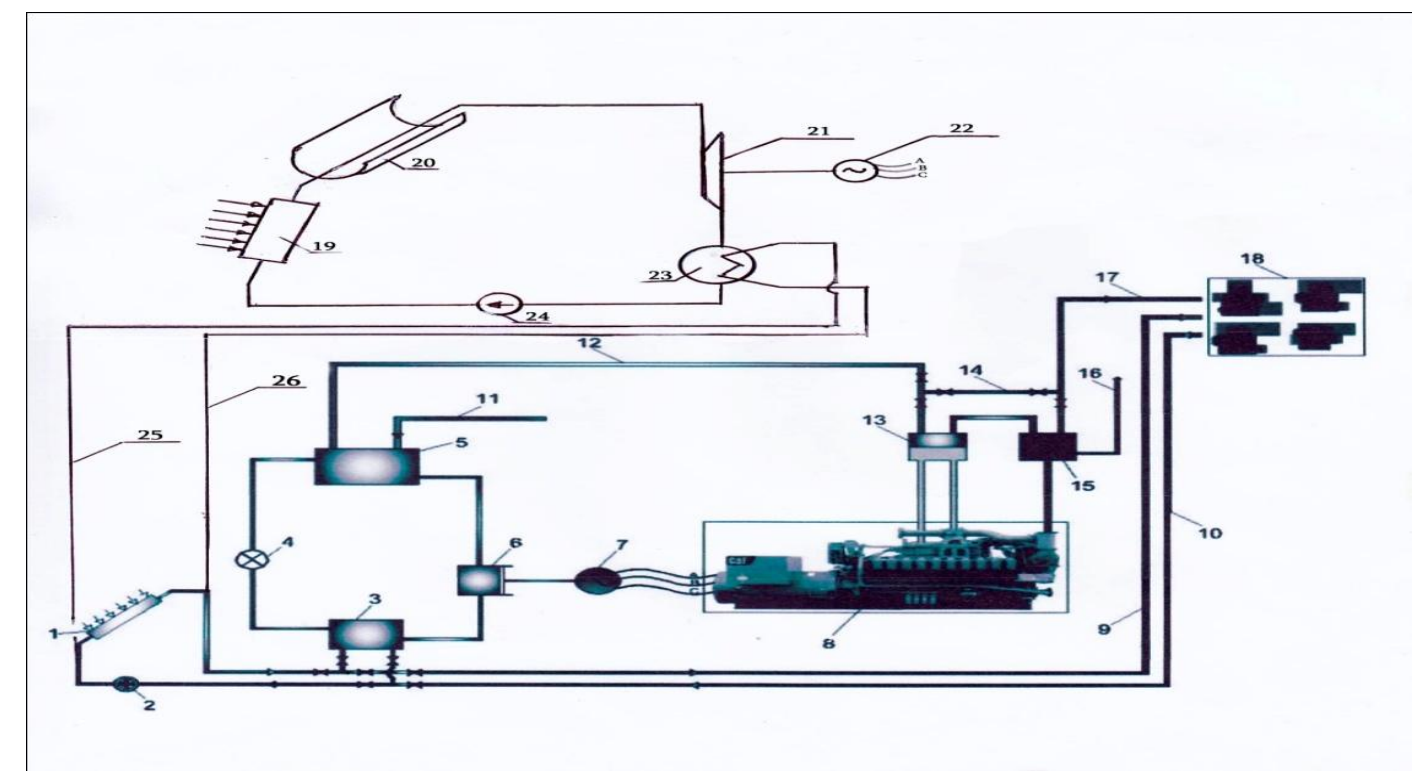

Pic. 1 Residential district heat, cold, electricity supply principal scheme in case when the source of heat, cold and electricity supply are gas cogeneration station and solar power plant that jointly carry out theheatpump power supply of the district. 1 , 19. Flat solar collector, 2. Heat pump circulator pump 3. evaporator, 4. Throttle valve, 5. Thickener, 6. Compressor, 7. Heat pumpelectromotor, 8. Gas cogeneration station, 9 and 10.Coldrunningbackbone supplier and return pipes, 11 and 12. Heat-running backbone supplier and return pipes, 14. Bypass line, 13 and 15. Heat - exchangers" "cooling liquid - water", "combustion gas - water", 16. Water pipe cold water, 17. Heat pipe backbone supplier pipes, 18. District buildings, 20. Vacuum reflector solar collector, 21. turboexpander, 22. Electric generator, 23. Solar power plant operating body condenser, 24. Operating body circulator pump, 25, 26. Condenser cooling circulating water supplier and return pipes Inclusion of the lowest price flat solar collector in the scheme, especially when it will operate in medium and low temperature domains, is due to low heat losses with reference to reflection and convective small heat flows. Characteristics of flat solar collector and other types of solar collectors are determined due to the given methods of [6], and the scheme operation, structure and calculationof methods and results are given in [7].:

\section{Methods}

As it is given in [6] the absorbed and useful heat energy of the solar collector is largely dependent on the thermal input and output temperatures, the various thermal losses. Each species has a certain density of sunshine radiation, which is indicated in their technical passport. 
Since large quantities of organic fuel are needed for microclimatic heat and cold supply systems in residential buildings, solar energy is of high importance for those countries that do not have their own organic fuels, including those for Armenia. That is why the use of energy-saving technologies in the heat and cold supply system in Armenia is an important issue in scientific research, among which one can also refer to the above mentioned scheme. Flat and vacuum reflector solar collectors are widely used as low temperature heat source for the purpose of providing heat in heat supply system. The main purpose of this article is the detailed study of rational use capacities of solar collectors for the needs of thermal energy supply system and solar power plants.For this purpose solar collectors' thermal energy conversion efficiency have been determined due to the following formula.

$\eta_{S K}=\eta_{o}-k_{1} \cdot \frac{\Delta T_{\text {mid. }}}{I_{c}}-k_{2} \cdot \frac{\Delta T_{\text {mid.. }}{ }^{2}}{I_{c}}$,

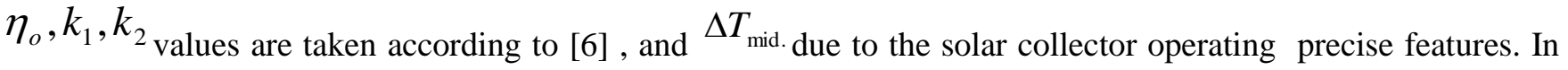

(1) $I_{c}$ - values are determined due to the climatic tables.

As it is shown in pic. 1, the required heat energy of the heat pump electric motor during daytime and favorable hours when there is sufficient sun radiation is produced in solar power plant and at night or at unfavorable hours or days from gas cogeneration station. Based on the above mentioned and the existing shortcomings in urban residential areas for thermal cold and electricity supply, when there is no need for a large electric capacity for solar power plant, it needs to meet mainly the needs of the heat pump electric drive, the above scheme is drawn up in Pic.1. In order to make it possible to use more simple and cheap solar concentrators namely flat solar collector, vacuum and vacuum reflected solar collectors there is a need to apply non-traditional low heat temperatures of operating body. On the other hand as a cosequence the use of low productivity turboexpanders for operating body expansion could be applied.

Due to the formula (1) and algorithm made in Excel, the thermal energy conversion eficiency coefficients for various types of solar collectors are determined in summer months. In the case of flat solar collectors the values conditionally turned to be negative because losses are greater than the radiation flow, that is, the above-mentioned temperature can not be reached during the other months of the year. In the example of vacuum solar collectors the values are 0,162 in January, 0,567 in July and 0,103 in December. This means that in the average seasonal conditions of the heat load of a residential area, for example $\sum Q_{\text {heatload }}^{\text {reg }}=4.5 \mathrm{MW}$, the average seasonal electric power of its electric motor in terms of MWwill be $1.5 \mathrm{MW}$, average seasonal conversion coefficient, in terms of the value of $\mu=3$. In these conditions when $\eta_{\text {steam loc. }}^{\text {SEP }}=0.8$,total average seasonal heat demand of the mentioned types of solar collectors will be $\sum Q_{H P}^{S C}=18,75 \mathrm{MW}$. In these conditions, in order to provide the heat demand of vacuum and vacuum reflected solar collectors $\left(\eta_{\mathrm{SC}}^{i}=0.291 ; 0.571 ; 0.251\right.$ ), the required surface according to the mentioned months will constitute

$$
\sum_{i=1,7,12} F_{S C}^{r e g}=565401,7 ; 57046,7 ; 1014412 \sum_{i=1,7,12} F_{S C}^{r e g}=314818,9 ; 56588,4 ; 416341 \quad \mathrm{~m} 2:
$$

This means that regional thermal heat, cold and electricity supply system for such operating body and climatic conditions of Erevan cannot operate around a year, as a consequence of extremely large surface of the area. Only in warm weather, that is from May to October, on average the local area of vacuum solar collector can compile $73077 \mathrm{~m} 2$ (provided it should not be abused, the given area should not be located in the suburbs the free lands should not be agricultural etc. )

There is also limitation for the application of solar plant as nowadays steam turbines with low parameters and small electric power are not produced. As it is shown in the scheme of pic. 1 the operating body ensuring the efficacy of solar power plant will operate in low potential domain, and the solar energy accumulation will be implemented gradually by heating, then evaporating and eventually overheating to the extent of the following formula $\Delta t_{\text {sup.heat }}$. It is assumed to use flat solar collector for heating (19), and vacuum and vacuum reflected 
solar collectors for evaporating and overheating (20). The extension of operating body will be notable as turboexpanders will be used.

Nowadays for the extension of operating body in gas supply systems and refrigeration techniques turbo-expanders with small capacity of radical and centrifugal action are produced, the usage of which in solar power plant system can be considered practical and perspective. As non-traditional operating body the technical opportunities of applying freons, butane, isobutane have been studied. R-22, R-142, R-114, R-113, R-113h, R-113 (when $\eta_{\text {det. }}=0.7$ ), R-134a freons were reviewed, when the heat of condensation of the used operating body in solar power plant is used as low temperature heat source for thermal plant, this means that the condenser of solar power plant can be combined with the vapor of thermal pump, as in cascade refrigerating machines. According to this the following terms have been adopted $t_{c o n d .}^{S E P}=t_{e v}^{H P}-3 \ldots 5^{\circ} C=0^{\circ} C$ as temperature of condensation, $t_{\mathrm{o}}^{\mathrm{SEP}}=30,40,40,50,60^{\circ} \mathrm{C}$ of evaporation, the coefficient of turbo-expander's extension $\eta_{\text {det. }}=0,8$, the size of overheating $\Delta t_{\text {sup.heat }}=0,10,20,30^{\circ} \mathrm{C}$

Based on the obtained results lines representing the functional connection (see pic.2) for the specified operating body were built $\eta_{S P S}^{\text {real. }}=f\left(t_{\text {cond }}^{S E P}\right)$, when the size of overheating is $\Delta t_{\text {sup.heat }}=0,10,20,30^{\circ} \mathrm{C}$. It can be concluded from the pic. 2 that in case of different levels of overheating of operating body, the real thermal energy conversion efficiency of solar power plant is different. As for realization of overheating the required heat can be obtained in accordance with the additional surface of vacuum solar collector, thus from the energetic point it will be appropriate $\Delta t_{\text {sup.heat }}=0^{\circ} \mathrm{C}$ version.

It is assumed from pic. 2 that $\mathrm{R}-113$ provides in this case more $\eta_{S P S}^{\text {real. }}=0.06 \ldots 0.15$. Such result in other $\Delta t_{\text {sup.heat }}$ conditions cannot be provided by any freones. However it is due to mention that $\mathrm{R}-113$ has practical difficulties or restrictions on application. This is the reason why this operating body has not been observed for another $\Delta t_{\text {sup.heat }}$. For other $\Delta t_{\text {sup.heat }} \mathrm{R}-142$ is considered more appropriate, as it has no restrictions in application, however nowadays turbo-expanders for thus operating body are not produced.

Investigations have shown that various factors such as the type of operating body, the temperature of its evaporating, the size of $\Delta t_{\text {sup.heat }}$ overheating have significant impact on $\eta_{S P S}^{\text {real. }}$.

In pic. $3 \mathrm{a}$ and $\mathrm{b}$ are shown curves expressing the functional connection $\eta_{S P S}^{\text {real. }}=f\left(t_{\text {cond. }}^{\text {SEP }}\right)$ for operating body of R114 and R22 (one of the axes represents the temperature of operating body in the output of vacuum and vacuum reflected solar collector $t_{\text {sup.heat }}=t_{e v .}+\Delta t_{\text {sup.heat }}$, as well as the customized expansion capability of $1 \mathrm{~kW}$ of turbo - expander, when $\eta_{\text {det. }}=0,7$ and the required surface of vacuum and vacuum reflected solar collectors in climatic conditions of Erevan.

It is derived from the pic.3 that for R114, when $t_{\text {sup.heat }}=60 \ldots 100^{\circ} \mathrm{C}$, the real thermal energy conversion efficiency of solar power plant is the biggest and $t_{\text {sup.heat }}=60^{\circ} \mathrm{C}$ is considered the most appropriate one due to the fact that thermal energy conversion efficiency has the biggest values and the required surface of vacuum and vacuum reflected solar collector is smaller.

This pattern is maintained also for $F_{\mathrm{VRSC}}, m^{2}$. For lower $t_{\text {sup.heat }}$ values the thermal energy conversion efficiency is of R22 is smaller, thus the $F_{\mathrm{VRSC}}, m^{2}$ is bigger. This means it is more convenient to use R114 in solar power plant, however turbo-expanders operating with such operating bodies are not produced nowadays. There are some operating with $\mathrm{R}-22$ but they are produced by non -i ndustrial scale.

Similar studies have been carried out for other freones, however the more expedient ones are R - 114 and R - 22 . 
The above mentioned refers to the $t_{c o n d .}^{S E P}=t_{e v .}^{H P}-3 \ldots 5^{\circ} C=0^{\circ} C$ when the solar power plant functions in association with thermal pump and its condenser is thermal pump's vapor, thus there is combination of condenser and vapor of thermal pump.

a.

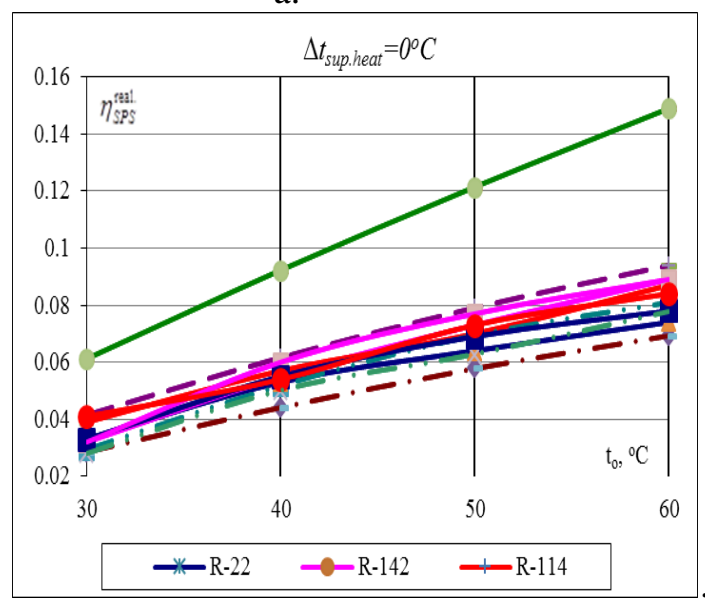

b.

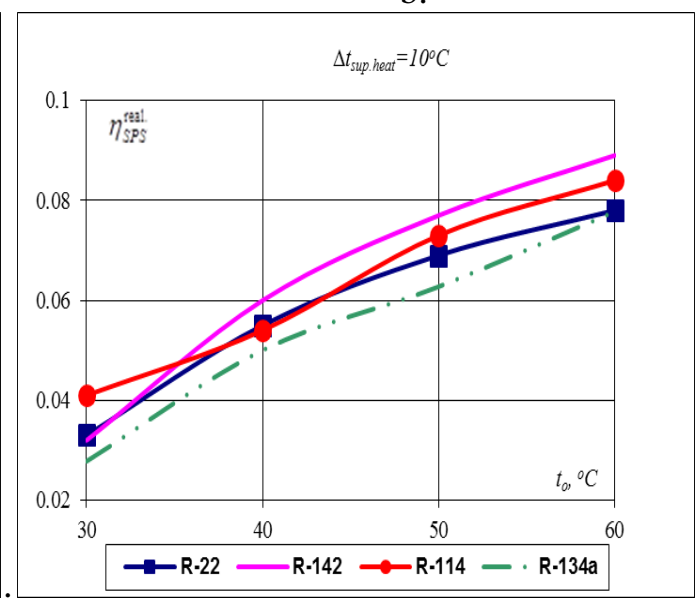

Pic. 2. The nature of the change in function $\eta_{S P S}^{\text {real. }}=f\left(t_{c o n d .}^{S E P}\right)$ when different working fluids, their condensation and evaporation temperatures.

As shown in pic.2 from a to $b$, when in case of different operating body we have a condition $t_{\text {cond. }}^{S E P}=t_{e v .}^{H P}-3 \ldots 5^{\circ} C=0^{\circ} C$ and $t_{\mathrm{o}}^{\mathrm{SEP}}=30,40,40,50,60^{\circ} C \eta_{\text {det. }}=0,8$, in case of solar power plant $\eta_{S P S}^{\text {real. }}=f\left(t_{\text {cond. }}^{\text {SEP }}\right)$ the functional link representing the changing patterns of lines on condition of $\Delta t_{\text {sup.heat }}=0,10,20,30^{\circ} C$ in accordance with the increase of $t_{\text {sup.heat }}$ the thermal energy conversion efficiency is decreased and equals to $\mathrm{R}-22$. The change of energy conversion efficiency of the latter occurs in opposite regularity. In parallel to the growth of $t_{\text {sup.heat }}$ it also grows, that is the overheating of this operating body is advisable unlike to $\mathrm{R}-114$.
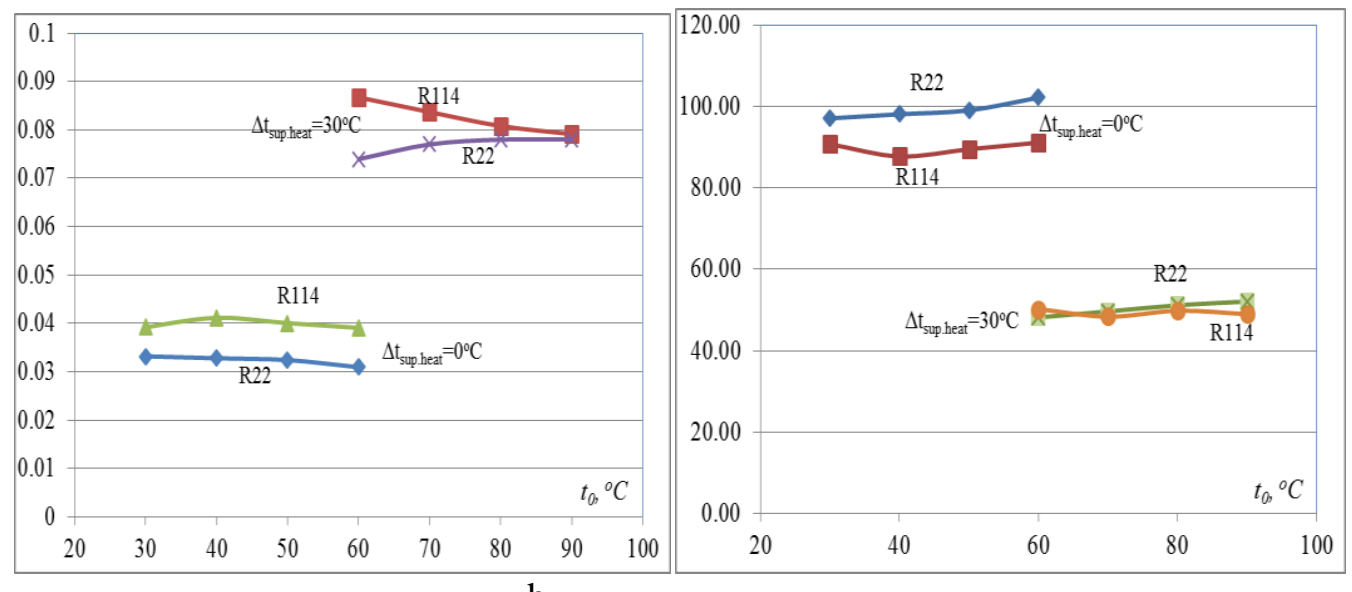

a. b.

In pic. $3 \mathrm{a}$ and $\mathrm{b}$ the operating bodies of R114 and R22 the customized expansion capability of $1 \mathrm{~kW}$ of turbo expander of solar power plant in climatic conditions of Erevan, when $\eta_{\text {det. }}=0.7$, the changes of real values of energy conversion efficiency and required surface of vacuum and vacuum reflected solar collector according to the formulae $t_{\text {sup.heat }}{ }^{o} C$ 
The results of the research revealed that the rational use of solar energy and in order to report it to operating body it is necessary to use the smallest specific evaporating thermal potentials (whereas the use of water is excluded) and the expansion of operating body must be proceeded in most possible realistic and specific conditions. Besides, there is a need to decrease the evaporating temperature of operating body in solar power plant system until degrees of $70 \ldots 80^{\circ} \mathrm{C}$.

Under such conditions the billing parameters of solar power station will be conditioned by the condensation temperature of operating body $T_{\text {cond. }}$, which is dependent on external air temperature $T_{\text {cond. }} t^{t_{\text {out. }}^{\text {int. }}}{ }^{+}+10^{\circ} \mathrm{C}$, as well as on the evaporating temperature of thermal pump $t_{c o n d .}^{S E P}=t_{e v .}^{H P}-3 \ldots 5^{\circ} \mathrm{C}=0^{\circ} \mathrm{C}$.

At the same time it follows that in order to organize the production of electricity from the solar power plant throughout day and year, there is a need of enormous surfaces of solar power station $50 \ldots 100 \mathrm{~m} 2 / 1 \mathrm{~kW}$. During unfavorable daytime and night hours, for different months of seasons the solar power plant must be equipped with thermal accumulating system, and for the heat cold and electricity suppliers regional uninterrupted functioning thermal and cold collectors are needed, electricity storing source as gas cogeneration station (see pic.1) or regional substations must be connected to one power system.

Nowadays specific attention is paid to the usage of natural refrigerants, as they comply with global warming and ozone-depleting regulatory requirements. That is the reason why the technical capability to use the isobautane $\mathrm{R}$ 600a has been observed, moreover nowadays appropriate turboexpanders for methane and $\mathrm{R}$ - 600a are produced in Russian Federation. Such is turbodetender of the type DGA, which can be operated with natural gas and isobautane in the conditions of $3 \ldots 1.5$ bar pressures and $2.162 \mathrm{~m} 3 / \mathrm{h}$ dimensional extraction, extensor's thermal energy conversion efficiency $\eta_{\text {det. }}^{i}=0.68$, the combined electric generator provides $34 \mathrm{~kW}$ electric power and variable electricity with the characteristics of $380 \mathrm{~V} / 3 \mathrm{~F} / 50 \mathrm{~Hz}$. For this purpose parameter calculations have been carried out of solar power plant, when isobautane (R - 600a) is considered as operating body, and the characteristic temperatures are $t_{\mathrm{ev} .}=80, t_{\text {sup.heat }}=85^{\circ} \mathrm{C}, t_{\text {cond. }}^{S E P}=t_{e v .}^{H P}-3 \ldots 5^{\circ} \mathrm{C}$. From the obtained results it is derived that in case of these specified parameters with the help of vacuum and vacuum reflected solar collectors during the process of heating the operating body it's energy conversion efficiency during cold months of the year will make 0.42 and 0.40 which is acceptable amount for solar collector, and the medium seasonal one will make 0.54. As the condensing temperature of operating body in solar power plant throughout the year, when vacuum and vacuum reflected solar collector stands as thermal source of solar power plant, is modified dependent on external air temperature, whereas there will be different heating, evaporating and overheating specific thermal levels of operating body used in solar power plant system.

As a result of decrease of medium temperature used in the process of solar collector's thermal energy conversion efficiency is also decreased and compiles about 0.15 and 0.10 for the January and December, and average seasonal is 0.406 . In the process of overheating process of operating body the thermal energy conversion efficiency of solar collector again due to the modification of average temperature will compile 0.12 and 0.078 , and average seasonal one will be 0.393 .

Using the $P-i$ diagram for operating system of R600a, the thermal levels of heat, evaporating and overheating have been carried out, when the mass consumption makes up $G=0.001_{\mathrm{kg}} / \mathrm{seq}$., as well as the required maximum area for the vacuum and vacuum reflected solar collector throughout the year. They will subsequently form for December $0.178,0.255,0.025 \mathrm{~kW}, 2.51,13.87,1.81 \mathrm{~m} 2$ : In the case of $\eta_{\text {det.. }}^{i}=0.68$ the real average seasonal work of operating body in turbo -expander will make $39.27 \mathrm{~kJ} / \mathrm{kg} \sum N_{\mathrm{SEP}}^{\text {gen.. }}=344 \mathrm{kWh} /$ year. The real average annual work, which will consume the circulator pump of the operating body, when $\eta_{\mathrm{p}}^{i}=0.6$ will make $3.57 \mathrm{~kJ} / \mathrm{kg} \sum N_{\mathrm{SEP}}^{\mathrm{p} \text {. }}=31,3 \mathrm{kWh} /$ year, and average annual efficient work will make $35.7 \mathrm{~kJ} / \mathrm{kg}$, $\sum N_{\mathrm{SEP}}^{\mathrm{useful}}=312,7$ will make 0.084 .

$\mathrm{kWh} /$ year. So the average annual thermal energy conversion efficiency of solar power plant 
Other temperature modes can also be observed, which is however beyond the scope of this article. The order of determination of enlarged economic indexes of solar power plant, its results, as well as the description of the work of the given scheme of picture 1 are represented in [7].

\section{Conclusions}

1. In the proposed technological scheme the condenser of solar power plant and evaporator of thermal pump are combined in order to increase the overall energetic efficiency of heat, cold and electricity suppliers, thus excluding the immediate need of solar collector during the functioning of thermal pump in accordance with the functioning of solar power plant.

2. Suggestions are made to use the smallest evaporation at a given temperature and the low evaporation temperature in solar power plant for the rational usage of solar energy and for reporting it to the operating body and as such to use freones, buthones, isobutanes etc.

3. In the above-mentioned conditions of operating bodies it is suggested to carry out their enlargement process in turbo - expanders.

4. In solar power plant the usage of freones as operating body the $\mathrm{R}-113(0.06 \ldots 0.15)$ provides more $\eta_{S P S}^{\text {real. }}$, though it has some difficulties and restrictions on practical application.

5. For freon R - 114, when $t_{\text {sup.heat }}=60 \ldots 100^{\circ} \mathrm{C}$ the real energy conversion efficiency of solar power plant is the biggest, the surface required by the vacuum and vacuum reflected solar collector is small.

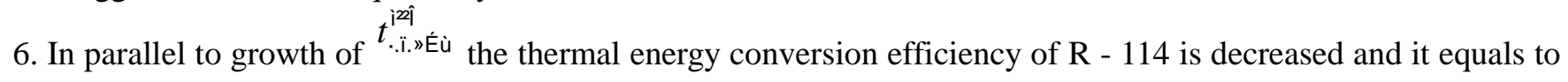
$\mathrm{R}-22$. The change of its thermal energy conversion efficiency goes in parallel with the growth of $t_{\text {sup.heat }}$, thus overheating of operating body is expedient, unlike the case of $\mathrm{R}-114$. Nowadays the production of turbo-expanders operating with such operating bodies is currently organized.

7. Throughout day and year in order organize the electricity production of solar power plant there is a need of enormous solar collector surfaces in accordance to the formula $50 \ldots 100 \mathrm{~m} 2 / 1 \mathrm{kw}$.

8. As nowadays there is a specific need of usage of natural refrigerants, as they are appropriate to global warming and ozone-depleting regulatory requirements;the technical opportunities of usage of isobutene R600a have been observed. In specific temperature domains for this operating body the average thermal energy conversion efficiency of solar power plant make up 0.084. Moreover nowadays for the enlargement of this operating body turbo-expanders are produced in Russia.

9. In the suggested scheme during non-solar time and days, when the application of solar power plant is inexpedient, it is intended to use cogeneration station, which will simultaneously provide part of the thermal need of the given region.

\section{References}

Petrosyan A. L. Application of air heat pumps for the heating of buildings. M, Energy saving №4-2015, p. $1-6$ The method of determining the main parameters for the design and operation of "heat pump - vertical soil heat exchanger", Mat. of XII international research and practice conference. 30.11 ... 07.12. 2016. Science and Education Ltd

Application of a heat pump for the residential buildings heat supply. -Moscow, Energy saving, №4-2015, p. 1 - 6 Petrosyan A. L., Barseghyan A. B. Prospects for the joint application of heat pumps and low-temperature solar collectors. Heat supply news. - Moscow 1, 2010, -p. 27-30.

Kotlaryov E. Yu., Anokhin A. B., How to choose the type of collector for the solar heating system? // "RainbowEngineering systems" LLC- http://alt.rkraft.ru/solnechnye-kollektory.html

Petrosyan A. L. Use of solar energy for heat supply of the urban area with the application of a heat pump and a solar pool. - Moscow: Energy security and Energy saving, 2, 2011, p.27-32

Petrosyan A. L. Use of solar energy in heat, cold and electricity supply systems for various consumers. -M.: Energy saving, 7, 2014, p. 66-72

Goossens, Ehren, "Chevron Uses Solar-Thermal Steam to Extract Oil in California", "Bloomberg", October 3, 2011. 